\title{
La experiencia del alto crecimiento en el Este de Asia. Balance a la luz de los nuevos desafíos
}

\section{Víctor López Villafañe}

En las últimas décadas, los estudios sobre el desarrollo han enfocado la experiencia de alto crecimiento de las economías del Este de Asia. Primero Japón, a partir de 1955, y luego Corea del Sur, Taiwán y Singapur, con sus modelos exportadores desde los años sesentas del siglo pasado, observaron elevadas tasas de crecimiento. Más recientemente, desde 1993, China desplegó un grandioso vuelo económico de altísimo crecimiento.

\section{INTRODUCCIÓN}

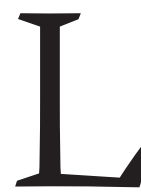

a literatura económica, que al principio estaba confinada a pocos especialistas en el área, devino en torrente cuando Japón emerge como una superpotencia económica, y entonces se habló del desafío a la hegemonía americana y del dominio para el siglo xxi de las economías de Asia Pacífico. La escuela más relevante que se formó para estudiar los factores impulsores del alto crecimiento es la del Developmental State o Estado desarrollista, que ponía el acento en el papel del Estado para impulsar el rápido y alto crecimiento.

El profesor Chalmers Johnson produjo el estudio clásico a partir del cual se estructuró esta escuela. ${ }^{1}$ Recordemos que era la época del inicio de los programas neoliberales en América Latina y que luego vendría el llamado «consenso de Washington», quizá por esta razón el Banco Mundial produjo un estudio en 1993 sobre el milagro económico del Este de Asia, donde reconocía la importancia de la intervención gubernamental en lo general; sin embargo, minimizaba y catalogaba de ineficaz a la política industrial y la promoción de las exportaciones. ${ }^{2}$

Como es usual, este fenómeno se abordó sin advertir que el alto crecimiento no era una receta universal, ni que su éxito podría engendrar nuevos problemas de carácter endógeno y, sobre todo, que el alto crecimiento no era un ciclo inmanente y perdurable ad infinitum. Tampoco se pensaba que se trataba de un proceso económico confinado en el tiempo y el espacio. Por ello, nadie pudo prever que Japón entraría en una larga deflación económica. Además, los propios países tuvieron cambios sociales que han venido a alterar la antigua 
fórmula de crecimiento. En especial, me refiero a los cambios demográficos en el área del Este de Asia, en especial Japón, pero también a otros como China.

En la actualidad, la inseguridad laboral, la reducción salarial y los problemas en las pensiones, además del aumento de las desigualdades, nos lleva a cuestionar el futuro de este modelo de desarrollo tan exitoso en el pasado y que desde nuestra perspectiva presenta formidables retos para las principales economías de la región.

\section{LOS SECRETOS DEL}

\section{ALTO CRECIMIENTO}

El alto crecimiento tuvo verificativo cuando el PIB creció a tasas promedio de más de $8 \%$ por etapas más o menos prolongadas y con caídas más o menos pasajeras. Así sucedió en Japón entre 1955 y 1980; Corea del Sur, 1963 y 1979, y en China, 1992 y 2007. En el caso de Japón, y en cierta medida Corea del Sur, después de elevadas tasas, sucedieron periodos de menor crecimiento en los noventa, con una crisis muy severa de deflación y bajo crecimiento en Corea en 1997 y, posteriormente, Japón. China ha podido mantener estas altas tasas de crecimiento hasta ahora, pero todo indica que ya ha iniciado el periodo de transición hacia tasas más moderadas. Lo que queremos plantear es el sentido histórico de este crecimiento a la luz de los nuevos problemas que han surgido. Por ejemplo, Japón se convirtió en una gran potencia económica y acumuló una riqueza financiera inconmensurable. Sin embargo, actualmente tiene una enorme deuda gubernamental y padece una deflación estructural, a lo que se agrega un gran cambio demográfico en el que los ciudadanos de más de sesenta años se convertirán en una gran masa poblacional. Y hay otros datos igual de importantes, como el de la reducción de los ingresos familiares y el aumento de los trabajadores temporales.

A continuación vamos a describir algunos elementos que han sido comunes a estos países, aunque cada uno de ellos muestra rasgos y características específicas. El primer factor común fue el trabajo barato. En la posguerra Japón tenía abundante mano de obra que fue contratada con salarios bajos. En Corea del Sur y China, especialmente, el trabajo barato de la clase obrera fue también un factor decisivo del ciclo de alto crecimiento. Se desarrollaron industrias intensivas en mano de obra barata y sólo cuando el factor trabajo empezó a escasear hubo necesidad de realizar aumentos de salarios. En Japón esta transición aconteció a mediados de los sesenta; en Corea del Sur a partir de 1977, y en China la escasez de mano de obra, sobre todo calificada, se ha ido agotando, dando lugar a los aumentos de salarios. Por esta razón se habla ya de que China está entrando en una nueva fase en la que deberá cambiar hacia industrias de alta tecnología. Además, en el caso de Japón, los sindicatos fueron aliados de las empresas; en Corea del Sur el Estado autoritario limitó y reprimió al movimiento obrero hasta que las luchas obreras hicieron posible el aumento de los salarios a mediados de la década de los ochenta. En el caso de China, la liberación de la mano de obra agrícola fue un factor determinante a partir de las reformas de 1978. Otro elemento a considerar en el caso chino es que los salarios industriales eran muy bajos en la era socialista precedente a las reformas económicas.

Otro factor importante consistió en el hecho de que contaron con monedas «baratas». El yen japonés se mantuvo en 360 por dólar desde la posguerra hasta 1972, valor que fue descendiendo paulatinamente hasta que a partir de 1985 el yen inició un periodo de revaluación que prácticamente anuló los antiguos beneficios para sus costes en la competencia mundial. El won coreano también fue fijado a una tasa moderada, pero tuvo fuertes devaluaciones a partir de 1964 en el momento de su viraje económico hacia las exportaciones. El yuan chino fue devaluado a principios de los noventa, con el fin deliberado de aumentar sus exportaciones y de
Como es usual, este fenómeno se abordó sin advertir que el alto crecimiento no era una receta universal, ni que su éxito podría engendrar nuevos problemas de carácter endógeno y, sobre todo, que el alto crecimiento no era un ciclo inmanente y perdurable ad infinitum. esta manera servir de palanca para lograr el altísimo crecimiento en esos años.

Un elemento central para el sostenimiento del alto crecimiento es que todas estas economías destinaron enormes flujos financieros para que las empresas tuvieran recursos de inversión prácticamente inagotables. En el caso japonés estos recursos financieros se otorgaron por conducto de los bancos pertenecientes a los grandes grupos empresariales. En el caso de Corea del Sur, el gobierno dirigió los recursos financieros por conducto de bancos nacionalizados y en China han sido los cuatro grandes bancos estatales 
los que han derramado una gigantesca cantidad de recursos de inversión para todas las empresas. Concomitante con lo anterior fue la programación de la política industrial hacia el sector de manufacturas rentables desde el punto de vista del mercado mundial. Este elemento que se inició en Japón, fue importante en la industrialización de toda la región, pues los cambios industriales y tecnológicos de Japón dejaban nuevas zonas industriales a los países que le seguían los pasos, como Corea del Sur. En China, la política industrial se aplicó geográficamente en las llamadas zonas económicas especiales y con la variante de utilizar abundante inversión extranjera.

El Developmental State que, según Chalmers Johnson, en Japón tenía como figura central a una burocracia no electa por los ciudadanos, inmune, al principio, a los intereses de los grandes consorcios, podía actuar de forma coordinada a favor de las políticas industriales. En Corea del Sur, después de 1953, la aristocracia terrateniente fue eliminada y el Estado podía también tener gran margen de maniobra en el campo industrial. En China, el Partido Comunista, sin ninguna oposición, ha podido realizar las políticas de mercado a partir de 1978.
No menos importante fue el papel jugado por la geopolítica, como contexto político-estratégico para el despegue y desarrollo de estas economías. La derrota de Japón en 1945 y el ascenso al poder de los comunistas en China marcaron el sello de las nuevas alianzas. Especialmente, Japón y Corea del Sur pasaron a ser aliados fundamentales para la contención del comunismo en Asia, y a cambio recibieron ayuda y acceso al mercado de Estados Unidos, lo cual fue un factor esencial para el éxito de los modelos exportadores. Japón se benefició de la guerra de Corea del Sur y este país, a su vez, de la guerra de Vietnam. La apertura de China, previa al deshielo de las relaciones con Estados Unidos, estuvo marcada por la victoria comunista en Vietnam y la necesidad de Estados Unidos de establecer una alianza con China para reconfigurar el poder americano ante los cambios mundiales en los setenta.

Otro de los puntos a destacar fue el relativo a cómo pudieron desarrollar su potencial tecnológico, así como generar empresas locales que con el tiempo se convirtieron en multinacionales. Todos estos países, también con matices, mantuvieron oleadas importantes de absorción

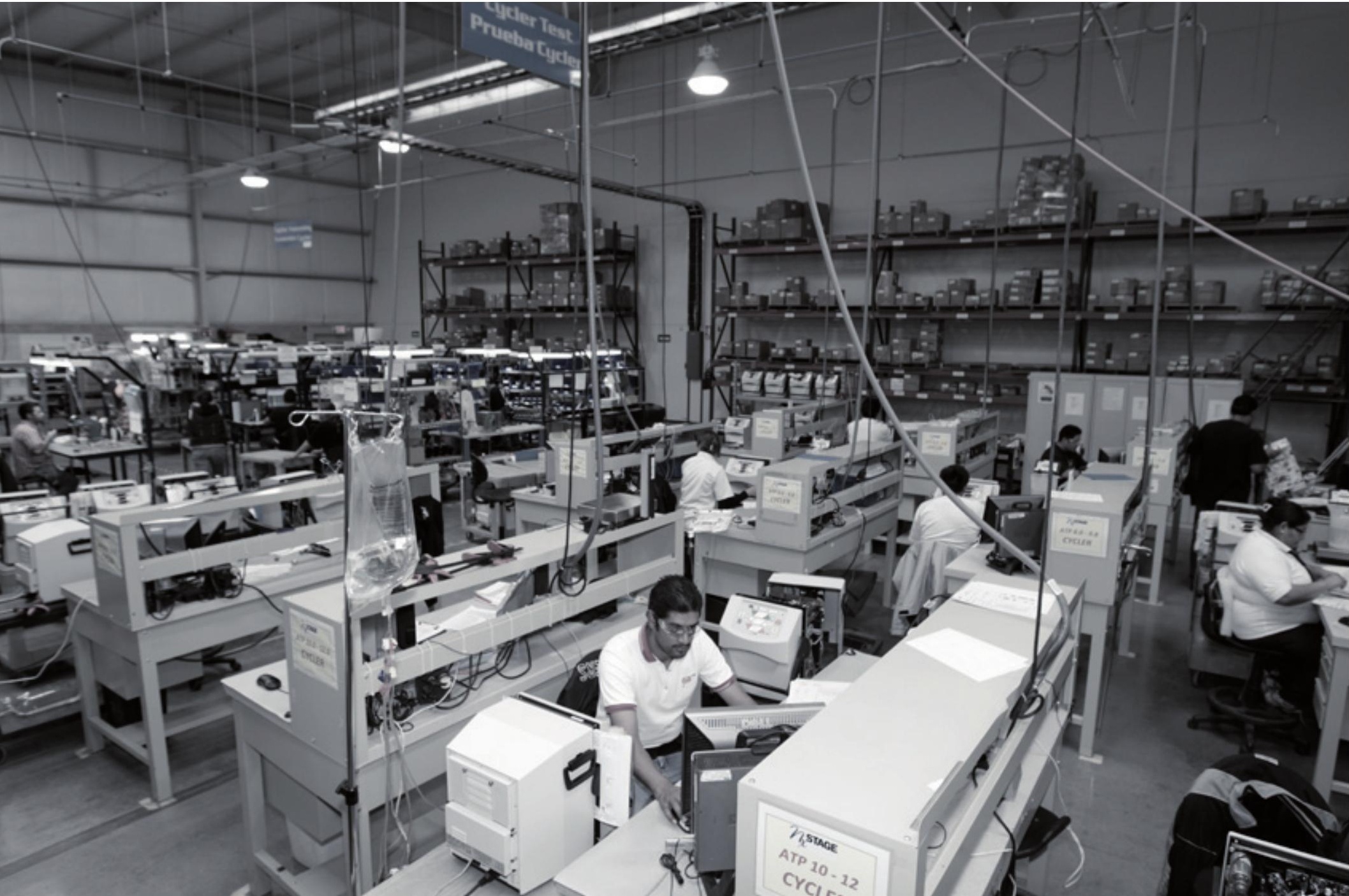


de nuevas tecnologías. Japón pudo desarrollar una cadena endógena de abastecimiento tecnológico para industrias intensivas, y luego transitó hacia la adquisición de nuevas tecnologías a partir de la posguerra y más tarde hacia la innovación en ramas como la automotriz, la electrónica y otras. En el caso de China, el desarrollo tecnológico proveniente de empresas extranjeras ha sido mucho mayor y se encuentra transitando hacia el desarrollo endógeno. Corea del Sur fue un caso intermedio, pero sus grandes empresas han sido muy agresivas en la competencia tecnológica desde hace varias décadas y eso explica, incluso, su apetito por capital y endeudamiento pasado.

Una de las cosas que sorprende de este modelo de alto crecimiento para el caso japonés y coreano es que las altas tasas de crecimiento sucedieron en un entorno que favoreció una relativa equidad en la distribución del ingreso. Los factores que empujaron en Japón hacia esta distribución del ingreso se pueden agrupar en los siguientes: ${ }^{3}$

1. Hubo casi pleno empleo, es decir, la tasa de desocupación fue menor a $2 \%$; prácticamente nadie, sólo los enfermos o discapacitados, se quedó al margen del empleo.

2. Entonces hubo escasez de trabajadores y se tenía que pagar más por las contrataciones. Así, los salarios aumentaron en esta época, independientemente de la productividad, empujada por los aumentos que ésta generaba.

3. El sistema de salarios por antigüedad evitaba desigualdades entre trabajadores al interior de las empresas.

4. Las ofensivas sindicales lograron aumentos importantes y éstos se trasladaron a otros sectores, como shadow price, es decir, toda la mano de obra japonesa quería y pedía su tajada en los aumentos de precios.

5. Los productos agrícolas y los servicios aumentaron su precio, independientemente de su productividad. A todo este proceso se le llamó «inflación por diferencia de productividad». El gobierno apoyó esta política dejando que los precios agrícolas aumentaran. Éste fue un mecanismo para realizar una distribución relativamente equitativa de los ingresos entre los diferentes estratos de la población.

6. Las pequeñas y medianas empresas también lograron al final del periodo aumentar su productividad, mejorando su entorno técnico y administrativo y con ello pudieron cerrar la brecha de salarios con las grandes empresas.

7. Según el profesor Nakamura, la distribución equitativa del ingreso originó que 95\% de la población japonesa se sintiera perteneciente a la clase media (encuesta 1960-1979).

\section{En Corea del Sur, los factores fueron: ${ }^{4}$}

1. Una política (1971) que favorece el ingreso familiar y las condiciones de vida en las zonas rurales, con apoyo al precio del arroz y control de precios en bienes básicos. Además, la rápida emigración del trabajo excedente hizo que aumentara el ingreso y disminuyera la brecha con el sector urbano.

2. Las oportunidades igualitarias de educación, sobre todo en primaria, que respaldó el desarrollo de industrias intensivas.

3. Los trabajos productivos para los desempleados y subempleados.

Por su parte, China tenía un sistema muy igualitario en la distribución de la riqueza nacional en la época de la estrategia socialista del auto-desarrollo, basado en comunas populares para el desarrollo del campo y empresas estatales en la industria. La sociedad china estaba rezagada industrial y tecnológicamente cuando se iniciaron las reformas en 1978, pero era una sociedad con rasgos igualitarios muy fuertes. Por ejemplo, el coeficiente Gini para los ingresos urbanos en 1980 fue de 0.16, mientras que para otros países en desarrollo el promedio era de 0.43. Este coeficiente en las zonas rurales fue en 1979 de 0.31, más bajo que el de otros países en desa-
El primer factor común fue el trabajo barato. Se desarrollaron industrias

intensivas en mano de obra barata y sólo cuando el factor trabajo empezó a escasear hubo necesidad de de salarios. realizar aumentos rrollo como India, de 0.34 . Pero esta igualdad ha ido desapareciendo dramáticamente en las dos últimas décadas. Así, en China, mientras más avanzaban las reformas de mercado, las desigualdades sociales han aflorado y esto significa una gran contradicción, ya que es formalmente un país «comunista». El coeficiente Gini ha crecido hasta 0.474 , esto significa que China es uno de los países más desiguales. El ingreso anual de los residentes urbanos es cuatro veces mayor que el de los residentes rurales. China se parece ahora más a un país latinoamericano del siglo $\mathrm{xx}$ que a un país «socialista». En Brasil y otros 
países de América Latina las desigualdades se han reducido, tanto por la aplicación de reformas institucionales como por acción del mercado, mientras que en China e India han venido creciendo. ${ }^{5}$

\section{Agotamiento del modelo de alto CRECIMIENTO Y NUEVOS DESAFÍOS: ¿DESENCANTO?}

Japón se encuentra en deflación permanente. En la actualidad, ha pasado de una gran riqueza financiera a una creciente deuda gubernamental cercana a $240 \%$ de su PIB. Las empresas están en constante presión por el alto valor del yen. Las tasas de interés, que están muy bajas, apoyan el desarrollo financiero externo a través de compra de bonos u otros instrumentos de mayores rendimientos. El cambio demográfico lo lleva ahora a ser una sociedad de adultos mayores. Además, bajo la presión de reducir costos, las empresas han tenidos que mantener bajas las pretensiones salariales. El ingreso familiar había caído 10\% entre 1997 y 2003, con más de $34 \%$ de trabajadores temporales (15\% en 1984). Así, la figura del trabajador permanente de por vida (SyushinKoyō) en las empresas japonesas es una reliquia del pasado. Por eso, en una reciente encuesta sobre las cosas que necesita hacer Japón, la profesora Noriko Hama propuso: 1) aumentar los salarios para alentar la demanda; 2) terminar con la política de cero interés, que puede ser buena para los deudores pero afecta a los retirados, y 3) deshacerse de la idea de que el crecimiento es una cura para todo y que aumentar los precios va a resolver todos los problemas de Japón. ${ }^{6}$

Los trabajadores temporales le conceden a las empresas japonesas una fuerza laboral flexible, que les ayuda a reducir los costos y así poder enfrentar la competencia global. Estos trabajadores reciben menos ingresos que los trabajadores permanentes, tienen menos beneficios y son despedidos en tiempos difíciles. Entre ellos se encuentran los trabajadores de tiempo parcial, los trabajadores «despachados o enviados» (hiyatoi haken) que reciben la mitad de salario de los permanentes por el mismo trabajo y horas laborales. El trabajador «despachado» es enviado a una empresa para cumplir obligaciones por las que ha recibido instrucciones de la empresa previamente, pero su contrato es con la compañía que lo ha despachado.
La clave está en que este trabajador recibe a diario instrucciones de la empresa en la que trabaja y no de la compañía que lo ha enviado a dicho trabajo. Luego vienen los trabajadores «subcontratados» por empresas subcontratistas. En el desastre nuclear de Fukushima, los héroes de la limpieza fueron en su mayoría este tipo de trabajadores. Al final de la lista están los trabajadores en «entrenamiento» (de origen chino, vietnamita y otras nacionalidades) que ganan cerca de 3.50 dólares la hora. El promedio salarial cayó en Japón cerca de $4 \%$ en 2009 , el tercer año de pérdidas salariales con una caída del salario real de 3\%. Todo este ejército de trabajadores no regulares tiene un impacto social muy fuerte en Japón, pues son un factor detrás de la caída de matrimonios y en las tasas de nacimientos. En el otro extremo, para los trabajadores permanentes, las responsabilidades y el volumen de trabajo se han incrementado.

Pasemos ahora al tema de la deflación histórica japonesa. La inercia de grandes volúmenes de inversión ha llevado a una espiral de sobreproducción y endeudamiento. Síndrome del pasado de alto crecimiento y la adicción a los préstamos para la producción. Según el profesor Motoshigue Itho, ${ }^{7}$ tenemos que examinar la brecha macroeconómica entre la oferta y la demanda en Japón para entender este proceso de deflación. Por más de 10 años, desde 1992 la oferta agregada era mayor a la demanda agregada creando lo que se puede llamar la brecha deflacionaria. Esta brecha significa que la economía tiene un exceso de capacidad que produce presiones deflacionarias y puede iniciar o perpetuar una tendencia a la baja de los precios. Los problemas de endeudamiento y las crisis financieras pueden ayudar a la formación de un proceso deflacionario, pero la causa fundamental en Japón de la caída de los precios sin lugar a dudas es la presencia a largo plazo de esta brecha deflacionaria.

Otro factor que ha hecho que esta brecha se mantenga por tanto tiempo ha sido el relacionado con el envejecimiento de la población y la declinación de la tasa de natalidad. Las personas adultas reducen sus gastos y la baja tasa de nacimientos es un problema grave para Japón, pues su fuerza laboral se está reduciendo y es una tendencia que va a permanecer.

El profesor Chalmers Johnson dice que la brecha entre la oferta y la demanda se formó cuando en lugar de expandir la demanda doméstica a partir de construir 


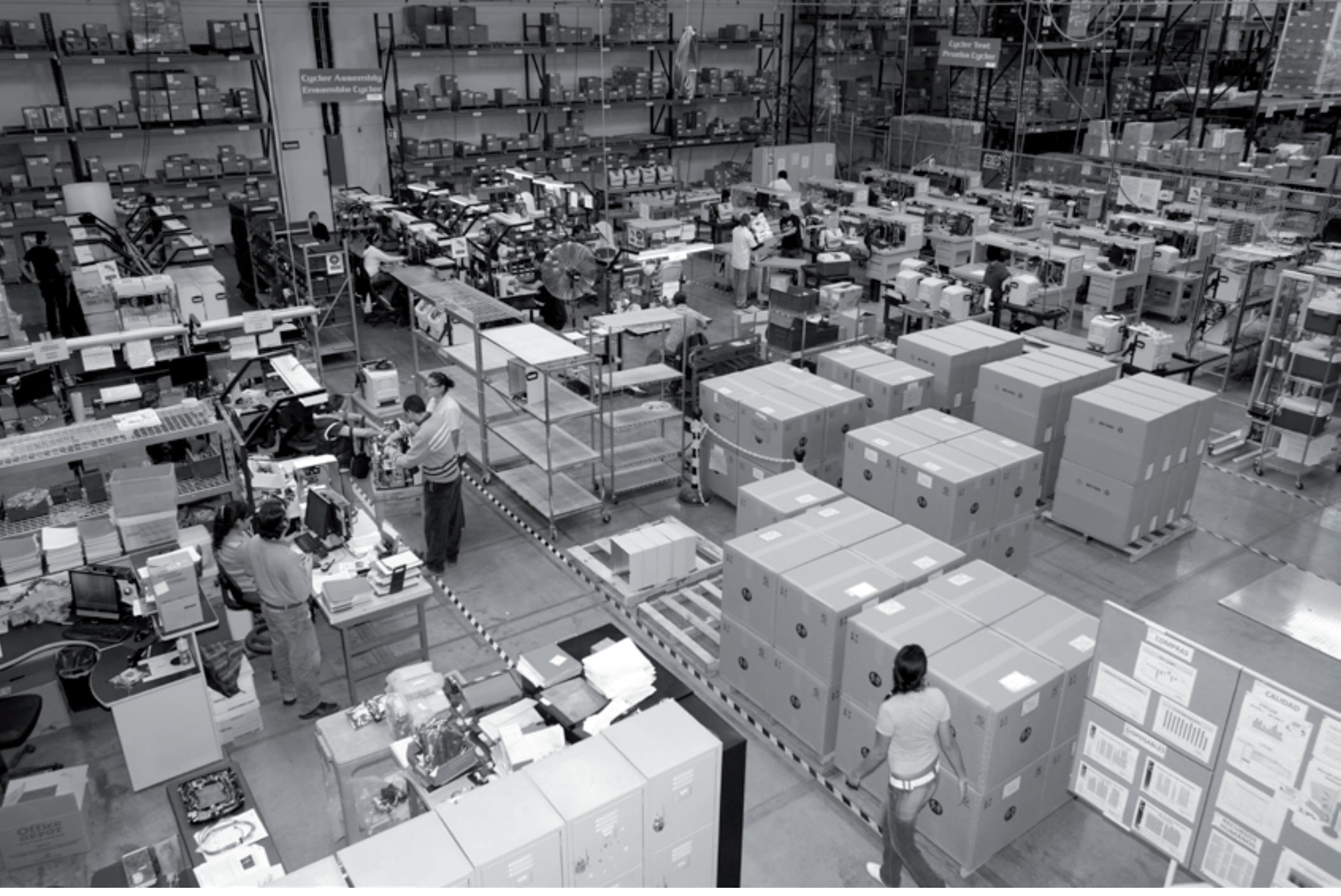

vivienda, hospitales y mejorar el transporte y la planeación urbana, la burocracia japonesa (MOF/Banco de Japón) redujo la tasa de interés para generar una onda de inversión. Entre 1986 y 1991, Japón invirtió 3.6 trillones de dólares en nuevas plantas y equipo e investigación, lo que produjo un exceso de capacidad productiva sin conexión con la demanda doméstica o internacional. La deflación en Japón fue la consecuencia política de la pérdida de la autonomía del Estado y la captura por parte de intereses especiales, bancos y empresas de construcción, de los órganos del Estado que en el pasado promovieron la política industrial. Por ejemplo, se gastaron trillones de dólares en toda una serie de proyectos de obras públicas innecesarias. Finalmente, para el profesor Johnson, el partido en el poder, el Partido Liberal Democrático (PLD), ha dejado de operar en su papel asignado dentro del Estado desarrollista y ha vuelto a caer dentro del marco de operación del viejo orden de la guerra fría, es decir, bajo el dominio de Estados Unidos. ${ }^{8}$

En Corea del Sur, la desigualdad social ha crecido y la economía se encuentra dominada por los grandes grupos industriales y financieros, los Chaebol. Los trabajado- res temporales son ahora un tercio de la fuerza laboral y sus ingresos representa $60 \%$ de lo que obtiene un trabajador permanente. De acuerdo con la Organización para la Cooperación y el Desarrollo Económicos (OCDE), el aumento de la desigualdad en Corea del Sur ha contribuido al aumento de la pobreza relativa a $14 \%$ en la mitad de la primera década del siglo xxi. La clase media coreana alcanzó su máximo en 1992, con 75.4\% y ha caído a $62.6 \%$ en 2009. El coeficiente Gini aumentó de 0.306 en 2006 a 0.314 en 2009. Todo lo anterior nos revela que el fin del alto crecimiento ha desembocado en este país en una serie de conmociones sociales.

El problema central en Corea del Sur está íntimamente ligado a la dinámica de los conglomerados gigantescos, Chaebol, que siempre han tenido un peso destacado y que hoy, después de la gran crisis económica coreana de 1997 y de la crisis mundial desde 2008, han vuelto a amasar una gran fortuna en detrimento de la sociedad. La pasada administración del presidente Lee Myung-bak fue caracterizada como promotora de los negocios de estos conglomerados. Para darnos una idea de su brutal concentración económica, cada año tienen 
ventas que representan más de $75 \%$ del PIB de Corea del Sur. Por eso, el tema central de las pasadas elecciones en diciembre en Corea del Sur tenía que ver con la «democratización económica», ya que el crecimiento moderado después de la crisis global de 2008 ha venido a ampliar la polarización social. Un segmento importante de la clase media coreana sufre de problemas de endeudamiento, los precios del sector inmobiliario están a la baja, hay desempleo fuerte en el sector de los jóvenes y muchas empresas micro han quebrado. Además, como en el caso de Japón, existe un cambio demográfico que hace que Corea del Sur tenga ahora una mayor población adulta que sigue aumentando y una baja en su tasa de natalidad.

Según el renombrado profesor coreano de la Universidad Nacional de Seúl, Byun Hyung-yoon, los privilegiados utilizan la excusa de que si los Chaebol son regulados, la economía no crecerá y se arruinará, pero esto lo que hace es en realidad monopolizar los beneficios del crecimiento económico. Por el contrario, justicia y distribución, desarrollo equilibrado y autosustentable deben ser el objetivo de esta democratización económica. Al igual que la profesora japonesa Noriko Hama, que hemos citado anteriormente, critica el concepto, muy arraigado en la sociedad, de que el crecimiento económico es lo más importante. Esto permite que los poderosos controlen a la sociedad y decidan la forma en que la riqueza debe ser distribuida. La premisa del crecimiento económico debe ser que todos en la sociedad puedan compartir los beneficios de este crecimiento. De este modo, para realizar una equitativa distribución de los beneficios, la concentración del poder económico de los Chaebol debe descentralizarse. La separación de su poder financiero e industrial es un requisito mínimo de esta reforma para regular a estos poderosos conglomerados. ${ }^{9}$

En China, el alto crecimiento muestra ya hoy grandes fisuras. Son tres los temas que van a gravitar en el futuro inmediato de China: 1) las enormes desigualdades sociales existentes y crecientes; 2) el cambio demográfico, y 3) el desastre ecológico. Los millonarios en China han venido aumentando (personas que tienen una riqueza de más de mil millones de dólares): en 2009 eran 129 y en 2011 pasaron a ser 271 (aunque se dice que muchas fortunas se encuentran «escondidas», así el número podría ser de 600 millonarios). Recientemente, el New York Times (25 de octubre, 2012) reportó que familiares del primer ministro Wen Jiabao habían amasado una fortuna de dos mil setecientos millones de dólares. Pero lo que más sorprende es que una tercera parte de los cincuenta más ricos son delegados en los órganos políticos más representativos o miembros del Partido Comunista. El salvaje alto crecimiento en China está desembocando en uno de los sistemas más inequitativos sobre la Tierra y ello ha presupuesto un enorme peso sobre la población china, que hoy se ve claramente en las continuas protestas que tienen lugar en prácticamente todo el territorio de ese país. Por esta razón, el Partido Comunista ha aumentado dramáticamente el presupuesto en el rubro de «mantenimiento del orden» (weiwen) en años recientes. En 2011, la Asamblea Popular aprobó un presupuesto de 95 mil millones de dólares en áreas relacionadas a la aplicación de la ley y el mantenimiento del orden.

El segundo punto que quisiera destacar es el cambio demográfico en China. La población entre 15 y 59 años - que puede considerarse el fundamento de la población en edad de trabajar- ha empezado a descender en términos absolutos desde 2010 y se dice que este grupo se reducirá en casi 30 millones de personas entre 2010 y 2020. Esto quiere decir que China va a enfrentar ya los problemas de una tasa descendente de su fuerza laboral. Todo esto tiene como fondo el debate sobre la caída de la oferta laboral frente a una demanda creciente de trabajadores, lo que produce cambios en toda la economía. Además, por la política de un solo hijo, la tasa de natalidad seguirá descendiendo y la brecha de género creará otro problema, al establecerse un desequilibrio entre la población masculina (que es mayor) y la femenina, lo que va a conducir a una reducción de los matrimonios y generará un nuevo problema social. En un interesante estudio, se afirma que la India tiene mejores perspectivas de jugar un papel destacado en el Asia Pacífico en este siglo XxI, basado en que su potencial demográfico será mejor que el de China y otros países. ${ }^{10}$

El problema ecológico es tremendo en China. Por la experiencia de otros países, como Japón mismo donde el alto desarrollo generó una contaminación bestial y dio lugar a grandes movimientos urbanos y a problemas de salud muy graves, como la llamada enfermedad de Minamata - nombre de la aldea donde las familias se enfermaron debido a que comían pescado contaminado de mercurio-, podemos decir que el desarrollo de 
China está ya en una frontera de enorme peligro por el desastre ecológico en el que se halla sumergido. Pekín y otras ciudades se están volviendo invivibles y el respirar buen aire tendrá un valor incalculable. Se han estado rebasando todas las normas y la población está expuesta a sufrir graves trastornos de salud. Éste será un peso que va a gravitar sobre la productividad de la fuerza laboral china. Además de los problemas del aire, está el del agua, la desertificación y otros que son obra directa de la corrupción en China, como la adulteración de productos alimenticios (caso de la leche materna) y bajos estándares en las regulaciones sanitarias.

Intereses creados, información asimétrica, abuso de poder y falta de respeto a la ley y regulaciones son todavía endémicas en el mercado chino y esto tiene efectos muy graves en la forma en que se conducen los procesos de mercado, así como las decisiones gubernamentales. Se requerirá una buena dosis de voluntad y coraje para llevar adelante las reformas políticas que serán necesarias para enfrentar todos estos desafíos y que son esenciales para instaurar un sistema empresarial moderno y sustentable en China. ${ }^{11}$

Terminamos diciendo que en estos países, en forma sucesiva, el alto crecimiento si bien sirvió para magnificar su potencial económico, ya que se convirtieron en potencias económicas, no dejó de traer nuevos y difíciles problemas, que ahora tendrán que resolver sin la posibilidad de volver a ese viejo ciclo económico de altas tasas de crecimiento. Este ciclo de alto crecimiento, sin embargo, ha dejado huellas indelebles -en el caso japonés, una larga deflación y agudizado la vida de la población trabajadora-. En el caso chino, el gran crecimiento está originando graves problemas sociales y ambientales. Quizá, como dice el profesor coreano Byun Hyungyoon, la justicia y la distribución deben ser los elementos que normen el desarrollo económico, cualquiera que sea la dimensión de éste. En el caso coreano, la crisis económica y financiera de 1997 introdujo una serie de políticas neoliberales que han provocado las desigualdades existentes actualmente en ese país, así como elevado la concentración económica de los grandes grupos empresariales. El desarrollo económico no debe ser sólo crecer a cantidades enormes de insumos de capital y trabajo, sino que debe estar orientado hacia la solución de los problemas sociales que tienen los países. Por ello, coincidimos con lo dicho por el profesor Chalmers Johnson cuando señaló que el aumento del poder económico de Japón debería haberse dirigido a resolver los problemas de la gente y no a aumentar la capacidad industrial inercialmente, ni en obras de infraestructura fastuosas desligadas de lo que se requería para el bienestar de la población. Así, podemos ver que el alto crecimiento en el Este de Asia nos deja una serie de lecciones importantes, después de años de mirarlos como un modelo a emular.

\section{Referencias}

1 Chalmers Johnson, «MITI and the Japanese Miracle», Stanford California, Stanford University Press, 1982.

2 Banco Mundial (1993), «The East Asian Miracle: Economic Growth and Public Policy», Nueva York, Oxford University.

3 Nakamura Takafusa, «Economía Japonesa. Estructura y desarrollo», México, El Colegio de México, 1990, pp. 285-292

4 Byung-Nak Song, «The Rise of The Korean Economy», Hong Kong, Oxford University Press, 1990, pp. 169-178.

5 Luis F. López-Calva y Nora Lustig (editores), «Declining Inequality in Latin America. A Decade of Progress?», Brookings Institution Press and UNDP Washington D.C., 2010.
6 Japan Times, january 13, 2013.

7 «Lessons of Japan's bubble economy», versión en inglés, Japan Echo Web, julio, 2010.

8 Chalmers Johnson, «Japanese Capitalism Revisited», JPRI Occasional Paper no. 22, agosto, 2001.

9 Entrevista al professor Byun Hyung-yoon bajo el título «Renowned profesor says growth isn't everything» en «the hankyoreh», 22 de septiembre, 2012.

10 Justin Logan, «China, America, and the Pivot to Asia», CATo Institute, Policy Analysis, no. 717, enero 8, 2013.

11 Ligang Song, «Unfinished reform threathens Chinese growth», East Asia Forum, enero 27, 2013. 